\title{
United Power Flow Algorithm for Transmission-Distribution joint system with Distributed Generations
}

\author{
Yirong $\mathrm{Su}^{1,}$, , Xingyue Jiang ${ }^{2, \mathrm{~b}^{*}}$, Yangyang $\mathrm{Xi}^{1, \text { a }}$ and Shuanghu Wang ${ }^{1, \mathrm{a}}$ \\ ${ }^{1}$ State Grid Electric Power Research Institute, Nanjing 211106, Jiangsu, China \\ ${ }^{2}$ Key Laboratory of Smart Grid of Ministry of Education, Tianjin University, Tianjin 300072, China \\ asu_yirong888@163.com, bxyjiang@tju.edu.cn
}

\begin{abstract}
Keywords: United power flow calculation, Transmission-distribution joint system, Distributed generations
\end{abstract}

Abstract. With increasing numbers of distributed generations (DGs) installed in distribution grids, the connection between transmission and distribution network becomes much stronger. Power flow calculation without consideration of transmission side may lead to inaccurate result, especially when penetration rate of DG are high. Thus a united power flow algorithm for transmission-distribution joint networks with DGs is put forward. Considering the electrical connection relationship and structural characteristics of these two grids, the whole system is firstly divided into transmission section and several distribution sections from boundary nodes. Appropriate power flow algorithm for each section is then chosen, and the interaction between transmission and distribution grids is also taken into account. The feasibility and effectiveness of this proposed method are testified by a transmission-distribution joint system with promising results.

\section{Introduction}

It is an inevitable trend that more and more DGs are connecting to distribution grids. This makes bi-direction of distribution power flow, that is, the direction of power flow is not only from the transmission side to the distribution side, but also from the distribution side to the transmission side. The nature of distribution network is altered from a passive network to an active one. The interdependence of transmission and distribution network has been increased, and the interaction between transmission and distribution network has become much more significant.

To investigate the impact of DGs on power system, present works are mainly focused on distribution side [1]-[3], which is true when penetration rate of DGs is low. When a large number of DGs are connecting to power grid, power flow calculation without consideration of transmission side may lead to technical issues [4].

In order to achieve accurate calculation result, both transmission and distribution network should be taken into account in one power flow calculation. Thus a united power flow method is proposed.

\section{DG models in power flow calculation}

As distributed generators are becoming prevalent in distribution systems, modeling them is an important task in power flow algorithm. The type of DG model is determined by operation modes and control characteristics of DG. Generally DGs can be classified as the following types[5].

PQ model. DGs whose active power and reactive power output can be controlled respectively are assigned to constant PQ models.

PV model. DGs with fixed active power output and voltage magnitude in power flow calculation can be treated as PV model.

PI model. DGs whose active power output and injection current are specified and fixed in power flow algorithm can be viewed as PI model.

PQ(V) model. The reactive power absorbed by asynchronous generator is determined by generator slip and voltage. Based on simplified steady-state model of asynchronous machine the reactive power absorbed by asynchronous generator can be calculated by a function of $\mathrm{Q}(\mathrm{V})$. 


\section{United power flow Algorithm}

General theories. A united power flow algorithm is adopted to accurately calculate the power flow of transmission-distribution joint system with DGs. In this method, the whole system is first divided into transmission section and a number of distribution sections from boundary nodes based on the differences between transmission and distribution networks. Therefore, the united power flow problem is divided into transmission power flow sub-problem and distribution power flow sub-problem. Appropriate algorithm is then selected to solve each sub-problem, and the interaction between transmission and distribution grids is taken into consideration as well.

Specifically, in the power flow calculation of transmission network, distribution grid is treated as a load with active and reactive power obtained from distribution power flow solution, while in the power flow calculation of a distribution network, transmission grid is viewed as an equivalent power source with a known voltage magnitude and angle acquired from transmission power flow solution. These two aspects of the power flow calculations are repeated and alternated until convergence is reached. The flow chart of this method is showed in Fig.1.

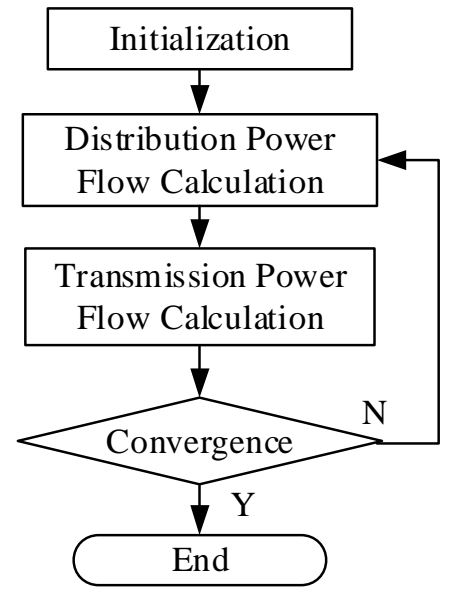

Fig.1 Flow chart of the united power flow algorithm

During distribution power flow calculation process, boundary node voltages are given and fixed, while active power and reactive power of root nodes are to be determined. Whereas, voltages of boundary nodes are to be calculated, while active power and reactive power of boundary nodes are unchanged in transmission power flow procedure. If any of node voltage mismatches is greater than tolerance, distribution power flow algorithm and transmission power flow algorithm are repeated until convergence criterion is met.

Improved Backward/ forward Sweep Algorithm. For the sake of power flow calculation of weakly meshed distribution network with DGs, a modified Backward/forward method is adopted. The distribution system is firstly converted to a radial network by breaking all loops at breakpoints. The branch currents interrupted by the creation of each breakpoint can be amended by current injections at its two end nodes. Using the multi-port compensation method described in [6], breakpoint currents are calculated by:

$$
\mathrm{Z} * \Delta \mathrm{I}=\Delta \mathrm{U}
$$

Where, $\Delta \mathrm{I}$ and $\Delta \mathrm{U}$ are the current injection and voltage mismatch of breakpoints respectively. $\mathrm{Z}$ is the Thevenin equivalence impedance seen form breakpoints.

As for DGs, several treatments need to be performed to analyze the impact of DGs on system network. The key point is to find out current injection of each DG.

The modified backward/forward power flow for distribution systems with DGs is an iterative process. Assuming the root bus is the slack bus with known voltage magnitude and angle, the iterative algorithm consists of four steps: 
1) Nodal current calculation: the injection currents from loads, DGs and breakpoints are calculated as a function of bus voltages.

2) Backward sweep to sum up branch currents: starting from the branches in the last layer and moving toward the root bus, branch currents are obtained in the backward sweep.

3) Forward sweep to update nodal voltages: starting from the branches connected to the root bus and moving toward the branches in the last layer, Nodal voltages are updated in the forward sweep.

4) Convergence check: After above three steps are executed in one iteration, the voltage mismatches at each bus and breakpoint are calculated separately. If any of the voltage mismatches is greater than convergence criterion, 1) - 3) steps are repeated until convergence is achieved.

\section{Numerical tests}

The proposed united power flow algorithm has been implemented in Microsoft Visual C++ programming language and tested on a transmission-distribution joint system shown in Fig.2. This joint system consists of three test systems -- one transmission system (system A) and two distribution systems (called system B and C respectively). System B and C are connected to system A at buses 2 and 4 respectively to form the joint system. The total loads of system B is $16.5+j 7.3 \mathrm{MVA}$, while the total loads of system $\mathrm{C}$ is $19.2+\mathrm{j} 8.5 \mathrm{MVA}$. The reference capacity of this joint system is 100MVA.

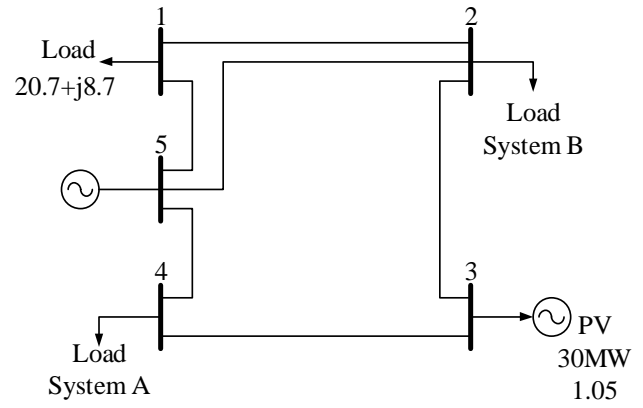

( a )

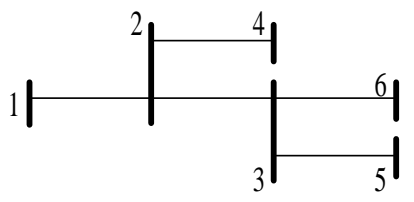

(b)

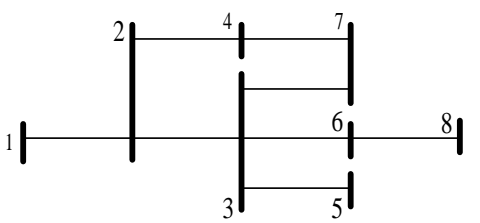

(c)

Fig.2 A joint test system: (a) five-bus transmission system; (b) six-bus radial distribution system;

(c) eight-bus weekly meshed distribution system

For testing purposes, DGs in PI and PV model are added to system B at buses 3 and 6 respectively, while DGs in PI, PQ(V) and PV model are introduced to system $\mathrm{C}$ at buses 4,5 and 8 respectively. The parameters of DGs are as follows:

PI model in system $\mathrm{B}$ and $\mathrm{C}: \mathrm{P}=2.0 \mathrm{MW}$ and $\mathrm{I}=20 \mathrm{~A}$;

$\mathrm{PV}$ model in system $\mathrm{B}$ and $\mathrm{C}: \mathrm{P}=5 \mathrm{MW}$

$\mathrm{PQ}(\mathrm{V})$ model in system $\mathrm{C}: \mathrm{P}=1.5 \mathrm{MW}, \mathrm{r}=0.0151 \Omega, \mathrm{x}_{6}=0.2714 \Omega, \mathrm{x}_{\mathrm{m}}=3.23 \Omega, \mathrm{x}_{\mathrm{c}}=1.55 \Omega$.

Newton-Raphson method is adopted to calculate power flow of transmission system when modified backward/forward sweep method is used to solve distribution power flow problem in the united algorithm. To validate this proposed algorithm, two cases are simulated. In case A, all DGs are removed from distribution networks. In case B, all DGs are added to distribution networks.

The united power flow algorithm converged in 2 iterations with an error tolerance of 0.01p.u. The convergence of each case is shown in Table.1. It is seen that the proposed solution algorithm is efficient. As the number of loops and PV nodes increases in case B, corresponding iteration does not increase dramatically.

Nodal voltage and complex Power of Root node and DGs are given in Table.2. "B-1" in Table.2 means node 1 in system $B$, and negative power compensation means the direction of nodal power is from DG to bus. It is shown that with the insertion of DGs, the local loads at system B and C are partially supplied by extra generation form DGs. This results in reduction of power supply from transmission grid.

In short, the proposed solution algorithm with efficiency and validity is capable of power flow calculation of transmission-distribution joint system with DGs. 
Table. 1 Convergence of each case

\begin{tabular}{|c|c|c|c|}
\hline Case Name & System Name & Iteration One & Iteration Two \\
\hline \multirow{3}{*}{ Case A } & System A & 2 & 2 \\
\cline { 2 - 4 } & System B & 2 & 2 \\
\cline { 2 - 4 } & System C & 2 & 2 \\
\hline \multirow{3}{*}{ Case B } & System A & 2 & 2 \\
\cline { 2 - 4 } & System B & 3 & 3 \\
\cline { 2 - 4 } & System C & 3 & 2 \\
\hline
\end{tabular}

Table.2 Nodal voltage and complex Power of Root node and DGs

\begin{tabular}{|c|c|c|c|}
\hline \multirow{2}{*}{ Case Name } & Node Name & Nodal voltage/p.u & $\begin{array}{c}\text { Complex Power } \\
\text { consumption/p.u }\end{array}$ \\
\hline \multirow{3}{*}{ Case A } & B - 1 & $1.018-\mathrm{j} 0.022$ & $0.168+\mathrm{j} 0.08$ \\
\hline \multirow{5}{*}{ Case B } & $\mathrm{C}-1$ & $1.029-\mathrm{j} 0.008$ & $0.201+\mathrm{j} 0.101$ \\
\cline { 2 - 4 } & $\mathrm{B}-1$ & $1.021-\mathrm{j} 0.002$ & $0.099+\mathrm{j} 0.043$ \\
\cline { 2 - 4 } & $\mathrm{B}-3$ & $1.021-\mathrm{j} 0.008$ & $-0.02-\mathrm{j} 0.004$ \\
\cline { 2 - 4 } & $\mathrm{B}-5$ & $1.05+\mathrm{j} 0.0$ & $-0.0506-\mathrm{j} 0.033$ \\
\cline { 2 - 4 } & $\mathrm{C}-1$ & $1.038+\mathrm{j} 0.009$ & $0.109+\mathrm{j} 0.061$ \\
\cline { 2 - 4 } & $\mathrm{C}-4$ & $1.018+\mathrm{j} 0.003$ & $-0.02-\mathrm{j} 0.004$ \\
\cline { 2 - 4 } & $\mathrm{C}-5$ & $1.015+\mathrm{j} 0.008$ & $-0.015+\mathrm{j} 0.007$ \\
\hline & $\mathrm{C}-6$ & $1.05-\mathrm{j} 0.004$ & $-0.0505-\mathrm{j} 0.032$ \\
\hline
\end{tabular}

\section{Summary}

A unified power flow method is developed for transmission-distribution joint system with DGs. DG models as well as the algorithm procedures are introduced. Test results of this algorithm application on a transmission-distribution joint system are presented and discussed. It is demonstrated that the proposed algorithm is available, efficient, and suitable for joint system with DGs.

\section{Acknowledgement}

This work is supported in part by the national science and technology support program(2013BAA01B04).

\section{References}

[1] Zhu Y,Tomsovic K.Adaptive power flow method for distribution systems with dispersed generation[J].IEEE Transactions on Power Delivery,2002,17(7):822-827.

[2] Moghaddas-Tafreshi, S.M., Elahe Mashhour. Distributed generation modeling for power flow studies and a three-phase unbalanced power flow solution for radial distribution systems considering distributed generation[J]. Electric Power Systems Research, 2009, 79: 680-686.

[3] Thomson M,Infield D G. Network Power-Flow Analysis for a High Penetration of Distributed Generation[J].IEEE Transactions on Power Systems,2007,22(3):1157-1162.

[4] Sun Hongbin, Guo Qinglai, Zhang Boming, et al. Master-Slave-Splitting Based Distributed Global Power Flow Method for Integrated Transmission and Distribution Analysis[J].IEEE TRANSACTIONS ON SMART GRID, 2015,6(3):1418-1492.

[5] Wang Shouxiang, Huang Lijuan. Unbalanced three-phase power flow calculation for distributed power generation system. Electric Power Automation Equipment, 2007, 27(8): 11-15. 
[6] Shimohammadi D, Hong H W, Semlyen A, et al. A compensation based power flow for weakly meshed distribution and transmission networks[J]. IEEE Trans on Power Systems, 1988, 3(2): 753-762. 